\title{
Positioning Control of an Underactuated Surface Vessel
}

\author{
Aníbal Matos and Nuno Cruz \\ Faculdade de Engenharia da Universidade do Porto \\ Instituto de Sistemas e Robótica - Porto \\ Rua Dr. Roberto Frias, 4200-465 Porto \\ Portugal \\ \{anibal,nacruz $\} @$ fe.up.pt
}

\begin{abstract}
This paper focuses on the positioning control of of a small size autonomous surface vessel (ASV) that can be used to carry a multitude of payload systems, including acoustic devices for underwater positioning and for communications with autonomous underwater vehicles. Its main motivation is the development of highly operational systems, by replacing typically moored support infrastructures with others that can dynamically position themselves.

This work covers the design of feedback control laws that assure that the underactuated surface vessel Zarco can keep its position even in the presence of water currents and wind, and without special sensors to estimate such disturbances. Experimental results showing the performance of the designed control laws are also shown.
\end{abstract}

\section{INTRODUCTION}

The last few years have witnessed the development of small autonomous boats, [1], [2], [3], as efficient tools for data collection missions, with an increasing number of applications. Some of these require the autonomous boat to hold its position at a given place.

The problem of actively controlling large vessels to keep their position and orientation has already been addressed in an extensive number of works. Typically, the positioning control is assured by a sufficiently large (and even redundant) number of thrusters that allow for the direct rejection of detected disturbances. However, this approach is not appropriate to small size ASVs, since these vessels usually possess only two thrusters and the integration of additional ones would increase the size, weight and complexity of these platforms.

The positioning control of underactuated vessels has already been addressed in several works, namely [4], [5]. In [4] it is shown that such vessels cannot be stabilized by a continuous state feedback of the state and a time-varying continuous feedback is proposed.

Our work proposes a discontinuous feedback controller to assure the dynamic positioning of a small size vessel, without the need of extra thrusters, and presents experimental results obtained with the ASV Zarco (fig. 1). The feedback controller takes into account environmental disturbances on the vehicle motion caused by water currents and wind.

The paper is organized as follows. Section II describes the ASV Zarco. Section III presents a dynamic model of the vehicle, while section IV addresses the kinematic and dynamic conditions that must be met when the vehicle is at rest. Section $\mathrm{V}$ discusses the proposed station keeping controller and, finally, in section VI, experimental results showing the behavior of the controller are presented.

\section{ZARCO ASV}

Zarco [6] is a small size craft designed to perform autonomous missions, mainly in rivers dams and estuarine environments. It was built with the double purpose of carrying general payload systems for collecting data in those environments, and of being a stationary or moving beacon for the navigation of underwater vehicles.

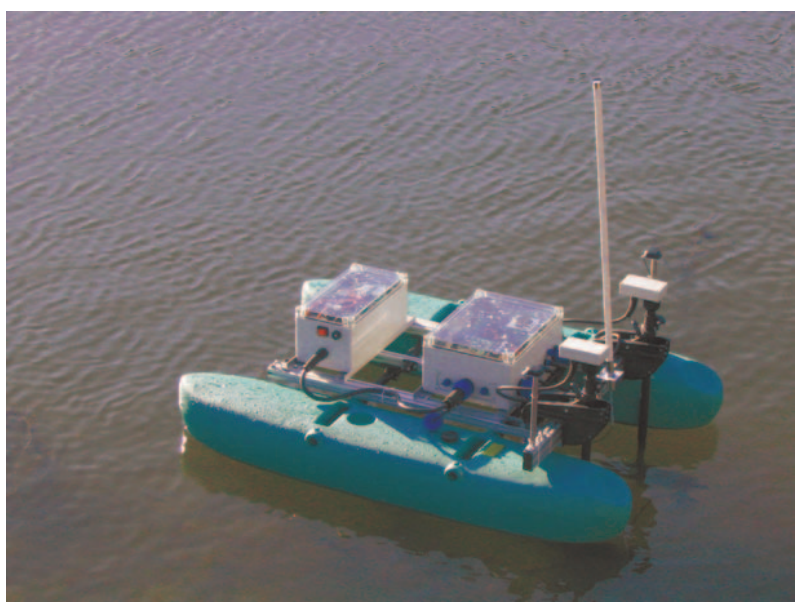

Fig. 1. Zarco ASV.

The vehicle is based on a catamaran type floating structure with 1.5 meters length and an overall width about 1 meter. In its basic configuration, Zarco weights a total of $50 \mathrm{~kg}$, and has an additional payload capacity of more than $25 \mathrm{~kg}$.

The vehicle is powered by two electric motors capable of delivering a total thrust of $250 \mathrm{~N}$, which allows for a maximum speed about 3 knots. The thrusters are located at the rear of the vehicle and their command allows the independent control of the vehicle forward and angular velocities.

A set of easy replaceable rechargeable lead acid batteries provide energy for more than 4 hours of continuous operation. The vehicle also carries an on-board computer responsible for 
the execution of autonomous or remotely controlled missions and for the storage of collected payload data.

The navigation system relies on data provided by a magnetic compass, an inertial measurement unit, a pair of L1 GPS receivers and a L1+L2 RTK GPS receiver. This set of sensors and devices allows the real time estimation of the vehicle position and attitude with high accuracies. When receiving DGPS corrections, the vehicle position accuracy is better than $5 \mathrm{~cm}$ for an update rate of $10 \mathrm{~Hz}$.

A WiFi link connects the boat to a shore station, allowing for the transmission of GPS base station data and for the supervision of the vehicle autonomous operations. In order to allow for different configurations, either in terms of floatation, propulsion, on-board energy, or payload sensors, the whole system was developed in a modular way, mainly using offthe-shelf components.

\section{VEHICLE MODEL}

The design of controllers for a given system is commonly based on a dynamic model that describes the relationship between the system inputs, outputs and internal variables. There are several ways to obtain a dynamic model for a given system, ranging from pure system identification techniques, which do not assume any a priori knowledge about the system, to the derivation of models based on well established laws that relate relevant variables.

\section{A. Vehicle dynamics}

The derivation of dynamic models for water vehicles usually follows the general approach presented in [7] to obtain a 6 DOF model for a partially submerged body. This approach is based on the laws of classical mechanics and can take into account the different kinds of forces and torques that act on a body or a set of bodies. In the general setting, the derived model depends on a large number of parameters since hydrodynamic terms are usually expanded in series.

In this work we considered a simplification of such general model by taking into account the vehicle symmetries and assuming that the vehicle moves horizontally with zero pitch and roll angles. Furthermore, we neglected both high order damping terms and hydrodynamic cross relations between the remaining modes (surge, sway and yaw). We also assumed that the body fixed frame is located at the centre of mass of the vehicle. Following these lines, we arrived at a 3 DOF model described by the following equations

$$
\begin{aligned}
& m(\dot{u}-v r)=X_{\dot{u}} \dot{u}+X_{u} u+X_{u|u|} u|u|+X_{a c t}+X_{w w} \\
& m(\dot{v}-u r)=Y_{\dot{v}} \dot{v}+Y_{v} v+Y_{v|v|} v|v|+Y_{w w} \\
& I_{z} \dot{r}=N_{\dot{r}} \dot{r}+N_{r} r+N_{r|r|} r|r|+N_{a c t}+N_{w w}
\end{aligned}
$$

where $u$ and $v$ are the surge and sway components of the vehicle velocity with respect to the water and $r$ is the vehicle yaw rate. Moreover, we also considered that the water current is constant, leading to external forces and torques that only depend on $u, v$ and $r$, on the disturbances caused by the wind and the waves $\left(X_{w w}, Y_{w w}, N_{w w}\right)$, and on the vehicle actuation $\left(X_{a c t}, N_{a c t}\right)$.
The actuation is related to the port and starboard thrusts ( $\left.F_{\text {port }}, F_{\text {starboard }}\right)$ according to

$$
\begin{aligned}
& X_{a c t}=F_{\text {port }}+F_{\text {starboard }} \\
& N_{\text {act }}=\left(F_{\text {port }}-F_{\text {starboard }}\right) \cdot \frac{b}{2}
\end{aligned}
$$

where $b$ is the distance between propellers axes.

\section{B. Kinematics}

To complete our model, it is also necessary to take into account the following kinematic relations

$$
\begin{aligned}
& \dot{x}=u \cos \psi-v \sin \psi+c_{x} \\
& \dot{y}=u \sin \psi+v \cos \psi+c_{y} \\
& \dot{\psi}=r
\end{aligned}
$$

where $c_{x}$ and $c_{y}$ are the north and east components of the water current in a fixed frame, $x$ and $y$ are the north and east coordinates of the vehicle position in an Earth fixed reference frame, and $\psi$ is the vehicle yaw relative to the local north.

\section{Wind disturbances}

The general modeling of wind and wave disturbances can be quite complex. Since we are considering an underactuated vehicle that cannot reject arbitrary disturbances, we will only take into account the low frequency disturbances caused by the wind. Furthermore, we will assume that the vehicle is both right-left and fore-aft symmetric. In such way, $N_{w w}$ will be zero and $X_{w w}$ and $Y_{w w}$ only depend on the forward and lateral components of the wind velocity with respect to the vehicle (which we will denote as $w_{u}$ and $w_{v}$ ) according to

$$
\begin{aligned}
& X_{w w}=C_{w u} w_{u}\left|w_{u}\right| \\
& Y_{w w}=C_{w v} w_{v}\left|w_{v}\right| .
\end{aligned}
$$

$w_{u}$ and $w_{v}$ are, obviously, related to the velocity and heading angle of the vehicle and to the water current by

$$
\begin{aligned}
& w_{u}=w_{x} \cos \psi+w_{y} \sin \psi-u-c_{x} \cos \psi-c_{y} \sin \psi \\
& w_{v}=-w_{x} \sin \psi+w_{y} \cos \psi-v+c_{x} \sin \psi-c_{y} \cos \psi
\end{aligned}
$$

where $w_{x}$ and $w_{y}$ are the north and east components of the wind velocity with respect to Earth. Furthermore, the coefficients $C_{w u}$ and $C_{w v}$ are given by

$$
\begin{aligned}
C_{w u} & =\frac{1}{2} \rho_{\mathrm{air}} c_{f} A_{f} \\
C_{w u} & =\frac{1}{2} \rho_{\mathrm{air}} c_{l} A_{l}
\end{aligned}
$$

where $\rho_{\text {air }}$ is the mass density of the air, $A_{f}$ and $A_{l}$ are, respectively, the frontal and lateral projected areas of the vehicle above the water and $c_{f}$ and $c_{l}$ are aerodynamic coefficients which we will assume to be unitary.

The two major disturbances that act on the vehicle when it is still at a given position are the hydrodynamic drag due to the water current and the aerodynamic drag due to the wind. It is, therefore, important to assess the relative influence of these two factors on the vehicle motion. This can be accomplished by determining the relationship between water 
current $c$ and wind speed $w$ that cause the same drag forces on the vehicle. Since the surge and sway drag coefficients are different, such relationships will differ for surge and sway motions. From equations (1)-(2) and (9)-(10) it is easily seen that the relationship between $c$ and $w$ will be

$$
0=X_{u} c+X_{u|u|} c^{2}+C_{w u} w^{2}
$$

for a surge motion, and

$$
0=Y_{v} c+Y_{v|v|} c^{2}+C_{w v} w^{2}
$$

for a sway motion.

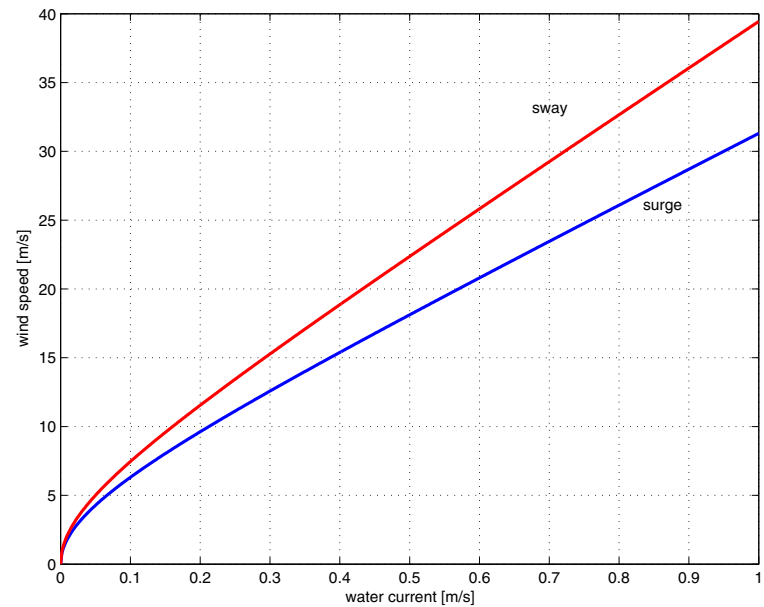

Fig. 2. Relationship between water current and wind speed.

Figure 2 shows both these relationships. As expected, the water current affects the vehicle motion much more than wind. For example, for a quite moderate water current, about $0.2 \mathrm{~m} / \mathrm{s}$, the equivalent wind speed is in the order of $10 \mathrm{~m} / \mathrm{s}$, which is already a relatively high value.

\section{REST CONDITIONS}

When the vehicle is at rest with respect to an Earth fixed frame, $\dot{x}=\dot{y}=0$ and the linear kinematic equations can be solved for $u$ and $v$ resulting in

$$
\begin{aligned}
& u=-c_{x} \cos \psi-c_{y} \sin \psi \\
& v=c_{x} \sin \psi-c_{y} \cos \psi .
\end{aligned}
$$

Moreover, since we are assuming that $N_{w w}=0$, the rest condition $\dot{\psi}=r=0$ together with (3) imply that $N_{a c t}=0$, meaning that at rest the differential actuation must be zero. On the other side, the conditions $\dot{u}=\dot{v}=0$ further imply that at rest the following relationships must hold

$$
\begin{aligned}
& 0=X_{u} u+X_{u|u|} u|u|+X_{a c t}+C_{w u} w_{u}\left|w_{u}\right| \\
& 0=Y_{v} v+Y_{v|v|} v|v|+C_{w v} w_{v}\left|w_{v}\right| .
\end{aligned}
$$

From this analysis, it is straightforward to conclude that, for given values of water current $\left(c_{x}, c_{y}\right)$ and wind speed $\left(w_{x}, w_{y}\right)$, equations (17)-(18) together with (20) can be used to determine the values of $u, v$ and $\psi$ when the vehicle is at rest. This is a set of three nonlinear equations that, in the general case, must be solved by numeric procedures. Equation (19) can afterwards be used to determine the common mode actuation $X_{a c t}$ required to put the vehicle at rest.

\section{A. No wind condition}

A simple, but nonetheless significant, condition arises when there is no wind. In fact, if $w_{x}=w_{y}=0$, equations (17)-(18) imply that $w_{u}=w_{v}=0$. In turn, equation (20) implies that $v=0$.

This result is just the obvious conclusion that if there is no wind, the vehicle at rest will be aligned (either forward or backward) with the water current, that is

$$
\psi=\arctan _{2}\left( \pm c_{y}, \pm c_{x}\right)
$$

with positive signs if $u<0$.

Furthermore, if the wind is aligned with the water current, a similar reasoning allows us to conclude that the vehicle at rest will stay aligned with both disturbances.

\section{B. Wind deviation}

When both water currents and wind are present, the orientation of the vehicle at rest will deviate from the direction of water current, in order to balance the wind drag by a sway velocity with respect to the water.

It is quite natural to expect that such deviation should increase as the wind disturbance gets stronger. Nonetheless, since the wind disturbes the ASV motion in a much lighter degree than water current does, it might be reasonable to predict that such wind induced deviation will be small for typical operating conditions.

To assess the validity of this reasoning, we estimated the deviation angle as a function of the wind speed for the case of a $90^{\circ}$ angle between water current and wind, and for three different values of the water current. The estimation was made by numerically solving equations (17), (18), and (20), and the obtained results are shown in fig. 3 .

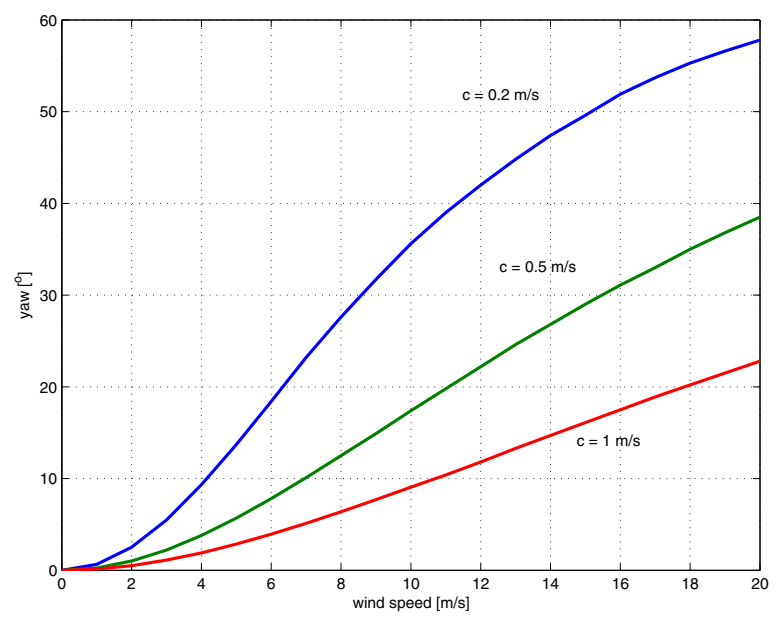

Fig. 3. Deviation angle due to wind. 
As expected, the deviation angles are higher for lower water currents, stressing that deviations ultimately depend on the relative weight of wind and water current. It is also clear that for moderate wind speeds, i.e., below $6 \mathrm{~m} / \mathrm{s}$, deviation angles are quite reduced, even for water currents as low as $0.2 \mathrm{~m} / \mathrm{s}$.

\section{Positioning Control}

Following the discussion on the previous sections, about the relative importance of the disturbances caused by the water current and the wind, we will neglect for now wind disturbances. Then, the ASV motion can be described by the following dynamic system

$$
\begin{aligned}
& m(\dot{u}-v r)=X_{\dot{u}} \dot{u}+X_{u} u+X_{u|u|} u|u|+X_{a c t} \\
& m(\dot{v}-u r)=Y_{\dot{v}} \dot{v}+Y_{v} v+Y_{v|v|} v|v| \\
& I_{z} \dot{r}=N_{\dot{r}} \dot{r}+N_{r} r+N_{r|r|} r|r|+N_{a c t} \\
& \dot{x}=u \cos \psi-v \sin \psi+c_{x} \\
& \dot{y}=u \sin \psi+v \cos \psi+c_{y} \\
& \dot{\psi}=r
\end{aligned}
$$

The station keeping problem consists in designing an appropriate controller that will drive the vehicle to a given point in the Earth fixed frame, which without any loss of generality we will take as the origin, that is, $x=y=0$. As we are not considering any direct measurement of the disturbances, the station keeping controller has to rely only on the data provided the navigation system, meaning that only $x, y, \psi$, and their time derivatives are available as inputs to the controller.

The controller proposed here has a double layer structure. The lower level is composed by to independent control loops, one giving the surge actuation $X_{a c t}$ and the other giving the yaw actuation $N_{a c t}$. The input references for both these control loops are computed by a top level guidance algorithm.

This algorithm follows a line-of-sight approach, with the necessary modifications to assure that in steady state the vehicle stays close to the origin with a reduced control effort. The input to the surge control loop is just a signed version of the distance of the vessel to the origin. The input to the yaw loop is the direction of the position vector of the vehicle with respect to the origin. This direction is taken in the forward or the backward way, depending on the current position and yaw angle of the vehicle. This results in a discontinuous dependence of the heading reference on the state variables $x, y$, and $\psi$, which is necessary for the convergence of the closed loop system. To avoid excessive chattering due such discontinuity, a dead zone, where the surge and yaw actions are keep constant, is considered. This dead zone is defined as the circle of radius $d_{m}$ in centered at the origin, and, in practice, its size should be related to the magnitude of the errors of the position estimates provided by the navigation system.

At each moment, the decision whether the vessel should approach the origin by a forward or a backward motion is based on the sign of the quantity

$$
s=-x \cos \psi-y \sin \psi \text {. }
$$

If $s \geq 0$, the vehicle should point to the origin and approach it with a forward motion. Otherwise, it should point against the origin and approach it with a backward motion.

According to the above discussion, the input $d_{0}$ to the surge loop is given by

$$
d_{0}= \begin{cases}0, & \text { if } d \leq d_{\text {min }} \\ d-d_{\text {min }}, & \text { if } d>d_{\text {min }} \wedge s \geq 0 \\ -d+d_{\text {min }}, & \text { if } d>d_{\text {min }} \wedge s<0\end{cases}
$$

where $d=\sqrt{x^{2}+y^{2}}$, and the input $\psi_{0}$ to the yaw control loop is obtained as

$$
\psi_{0}= \begin{cases}\psi, & \text { if } d \leq d_{\text {min }} \\ \arctan _{2}(-y,-x), & \text { if } d>d_{\text {min }} \wedge s \geq 0 \\ \arctan _{2}(y, x), & \text { if } d>d_{\text {min }} \wedge s<0\end{cases}
$$

The lower level control loops employ linear proportional plus integral controllers to determine the longitudinal and angular commands according to

$$
\begin{aligned}
& X_{a c t}=k_{x p} d_{0}+k_{x i} \int d_{0} d t \\
& N_{a c t}=k_{n p}\left(\psi_{0}-\psi\right)+k_{n i} \int\left(\psi_{0}-\psi\right) d t
\end{aligned}
$$

When the vehicle is inside the dead zone, $d_{0}=0$ and $\psi_{0}=\psi$, meaning that during such intervals $X_{a c t}$ and $N_{a c t}$ will remain constant. In steady state, $X_{a c t}$ will be the force required to balance the drag caused by the water current, and $N_{\text {act }}$ should be zero, therefore making the integral term in (32) unnecessary. Nonetheless, minor assymetries in the hull or between the thrusters might cause un-modeled yaw torques which will only be rejected by such integral term.

\section{EXPERIMENTAL RESULTS}

The station keeping controller proposed here has already been tested with success in several field experiments. These experiments were conducted in the Douro river, near Porto, Portugal, under different environmental conditions, but typically with significant water currents, due to the river flow.

To illustrate the performance of the station keeping controller, we next present the results of one of these experiments, conducted with a water current about $0.4 \mathrm{~m} / \mathrm{s}$ with a direction of $315^{\circ}$. In this case $d_{\min }$ as set to $0.3 \mathrm{~m}$, which was found to be a good compromise between station keeping accuracy and control effort.

Figures 4 and 5 present relevant data from one the experiments. The vehicle position stabilizes at a distance to the target close to $d_{\min }$ and stays approximately aligned with the current.

Figure 6 shows the trajectory and heading of the vehicle for this experiment. The ASV starts turning towards the target and, at the same time, its distance to the target decreases, mainly due to the water current at the beginning. After a certain time, the vehicle becomes aligned with the current and then makes the final approach to the target. Figure 7 shows both the linear and the angular actuation of the vehicle. After reaching the dead zone for the first time, both $X_{a c t}$ and $N_{a c t}$ are kept 


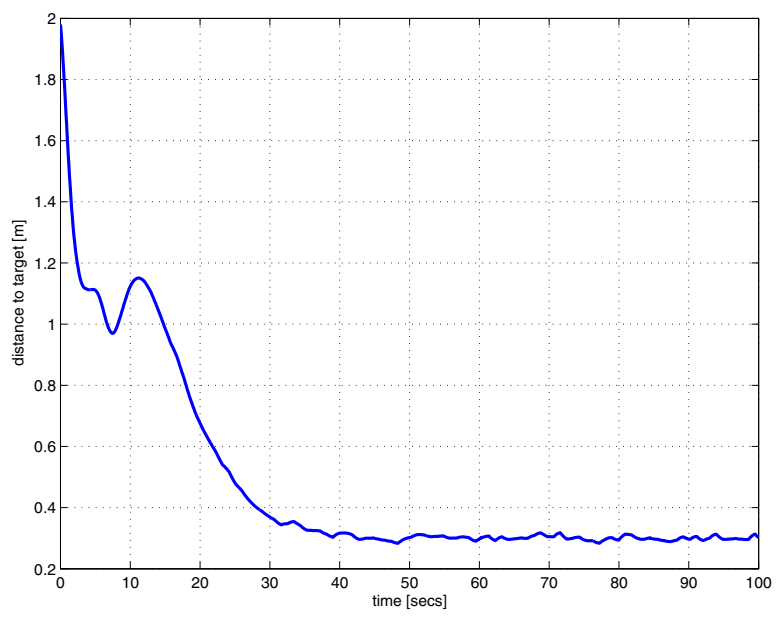

Fig. 4. Distance to target position.

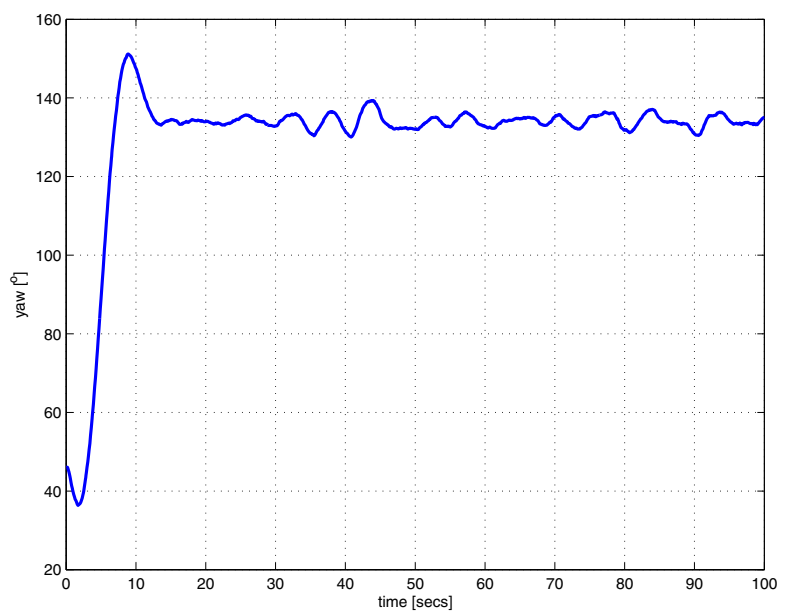

Fig. 5. ASV heading.

constant, except for the small amounts of time required to make the vehicle return to the dead zone.

\section{CONCLUSIONS}

The station keeping controller proposed here in based on a discontinuous feedback law and allows the stabilization of an underactuated vessel subject to environmental disturbances. It should be noted that this controller relies only on data provided by the vehicle navigation system, avoiding the estimation of water currents or wind speed and direction. This is a major advantage since the target platform in a small size ASV, where onboard space and energy are always at premium.

The results obtained in operational scenarios validate the approach used and the implementation of this positioning control system on the Zarco on-board computer already allowed the use of this ASV in new applications.

\section{ACKNOWLEDGMENT}

This work was supported by the project INCORP - Improved Navigation with Cooperative Robotic Platforms -

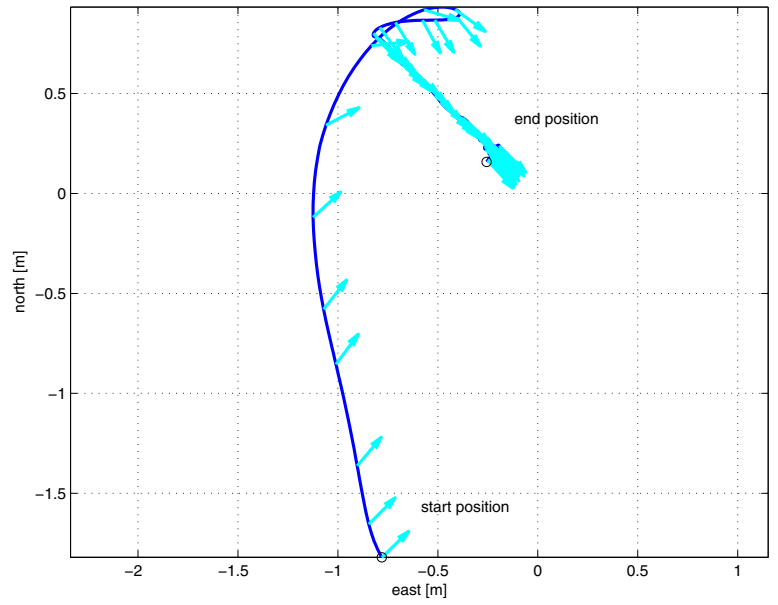

Fig. 6. ASV trajectory and heading.

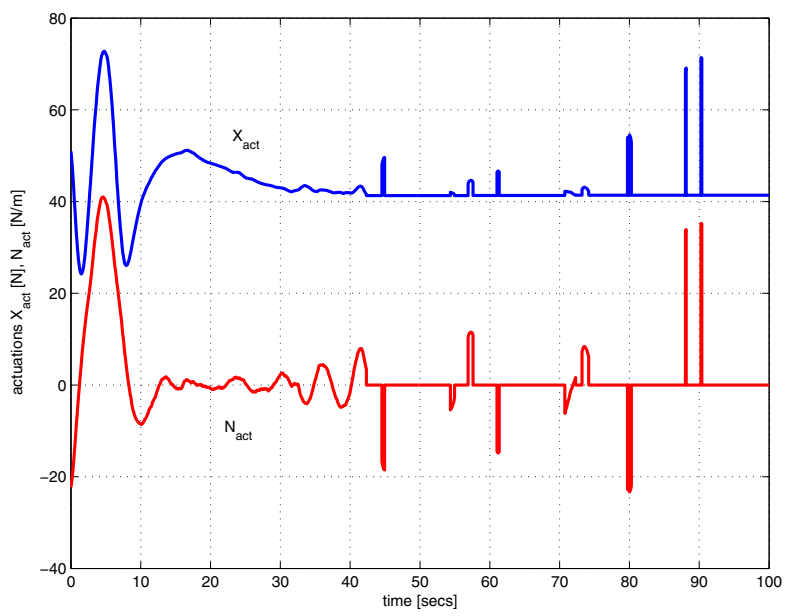

Fig. 7. ASV actuation.

funded by FCT under Programa POSC (ref. POSC/EEASRI/59963/2004).

\section{REFERENCES}

[1] J. Manley et al, "Applying AUV Lessons and Technologies to Autonomous Surface Craft Development”, Procedings of Oceans'01, USA, 2001.

[2] A. Pascoal et al, "Robotic Ocean Vehicles for Marine Science Applications: the European ASIMOV Project", Proceedings of IAV'01, Japan, 2001.

[3] M. Caccia et al, "Design and preliminary sea trials of Sesamo: an autonomous surface vessel for the study and characterization of the air-sea interface", Tech. Rep. Rob-SESAMO-03-01, CNR-IAN, 2003.

[4] K. Pettersen, T. Fossen, "Underactuated Dynamic Positioning of a ShipExperimental Results", IEEE Trans. Control Systems Technology, vol. 8, no. 5, Sep. 2000.

[5] A. Pereira, J. Das, G. Sukhatme, "An Experimental Study of Station Keeping on an Underactuated ASV", Procedings of ICRA'2008, Pasadena, CA, May, 2008.

[6] N. Cruz, A. Matos, S. Cunha, S. Silva, "Zarco - An autonomous craft for underwater surveys", Proceedings of Geomatica'2007, Barcelona, 2007.

[7] Thor I. Fossen, "Guidance and Control of Ocean Vehicles", John Wiley \& Sons Ltd., 1994. 